Japanese Psychological Research

1971, Vol. 13, No. 4, 183-191

\title{
A COMPARISON OF SIMILARITY SPACE AND SEMANTIC SPACE OF RANDOM SHAPES
}

\author{
TADASHI KIKUCHI ${ }^{1}$ \\ Tokyo University of Education
}

\begin{abstract}
Two experiments were run for 25 randomly generated shapes varying in the number of turns. Similarity judgments, made by 36 undergraduate students, were scaled using non-metric multidimensional scaling technique. As the results, 3 dimensions were obtained, representing dimensions of compactness, complexity, and symmetry. As a second experiment, judgments were made on 30 semantic differential scales by 40 undergraduate students. As the results of analysis, 3 factors were extracted, representing dimensions of activity, potency, and evaluation. These solutions were compared using canonical correlation method, which yielded only one significant relation between them. The total variance shared by the two spaces was only $22.1 \%$.
\end{abstract}

The present study is designed to contribute to the development of perception of forms. There are enormous variations among shapes due to the multidimensionality of forms. Nevertheless, the observers seem to be easy to judge or to discriminate the forms in any given tasks. Many studies of form perception have shown that the observers used a variety of attributes of shapes such as compactness, dispersion, jaggedness, elongation, symmetry, complexity, etc. in the various tasks (Aiken \& Brown, 1969a, b, c; Brown \& Andrews, 1968; Behrman \& Brown, 1968; Thomas, 1968). The observers, however, do not use all the attributes of shapes, but they use a limited number of the attributes of shapes. They seem to give selective

1 This study was conducted under the direction of Takayoshi Kaneko of Tokyo University of Education, to whom the author would like to express his sincere gratitude for valuable suggestions, encouragement in preparing the manuscript, and for a critical reading of the manuscript. The author indebted to Takayuki Mori, Chukyo University, for many suggestions. Thanks are also due to Akira Watanabe of NHK Broadcasting Science Research Laboratories who graciously offered facilities for this study. Finally, the author owes a special debt of gratitude to Hoben Thomas of Pennsylvania State University who was willing to send him Kruskal's program. attention to some attributes of shapes. The process of selection will be performed in a flexible way according to the tasks.

It would be of some interest to know what kind of the attributes of shapes are used in the similarity judgments and the semantic judgments and whether the dimensional structures of these psychological spaces of shapes are highly similar, using a multidimensional scaling techniques.

It has been suggested that multidimensional scaling techniques are useful tools to study the perceptual spaces of the shapes (Attneave \& Arnoult, 1956). Kruskal's non-metric scaling method (Kruskal, 1964a, b) has several advantages. This method provides general Minkowski $r$-metric solutions and enable us to compare the solutions each other in order to find an appropriate spatial presentation. Since there is an evidence that both metric and non-metric multidimensional scaling methods define almost same spaces (Behrman \& Brown, 1968), Kruskal's method will be used to analyze a similarity data in this study.

In the present study, the dimensional structure underlying similarity of the random shapes was first investigated. The second purpose was to analyze the affective 
properties of randomly generated shapes and then to discuss the possible relationship between the two spaces.

\section{EXPERIMENT I}

\section{Method}

Subjects Ss were 36 undergraduate students, 19 males and 17 females, of Chiba University. Of these, 26 were selected from the pool of students in an introductory psychology course (Group I) while the remaining 10 were students majoring in psychology (Group II).

Stimuli Stimuli used here were 25 random shapes, which were generated in a $100 \times 100$ unit matrix by Method I of Attneave and Arnoult (1965). As Fig. 1 shows, Stimuli 1-5 had 4 turns ; Stimuli 6-10, 8 turns ; Stimuli 1115, 12 turns; Stimuli 16-20, 16 turns; Stimuli 21-25, 20 turns. Each of the possible pairs of these Stimuli was photographed, and mounted as slides. The stimulus shapes were projected

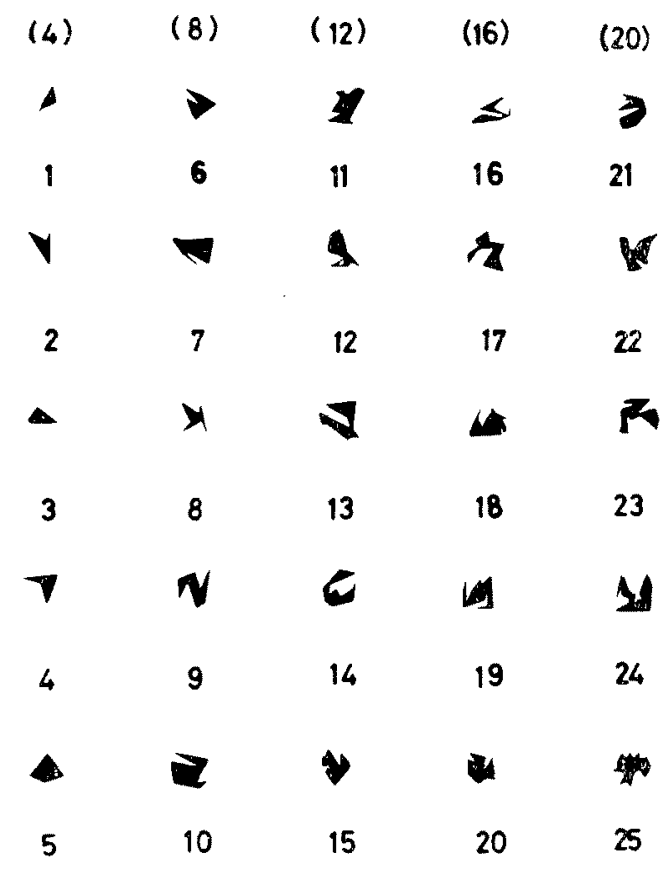

Fig. 1. The stimulus shapes used, Stimuli $1-5$ have 4 turns; Stimuli 6-10, 8 turns; Stimuli 1115, 12 turns; Stimuli 16-20, 16 turns; Stimuli $21-$ 25, 20 turns. onto a screen in a dark room. The sizes of the shapes were different each other. The smallest one was about $14 \mathrm{~cm} \times 20 \mathrm{~cm}$ on the screen, and the largest one was about $27 \mathrm{~cm} \times$ $27 \mathrm{~cm}$. They appeared black on a white background.

Procedure The pairs of stimuli were presented to $S$ s about $8 \mathrm{sec}$ per slicle. This viewing time was found in a preliminary experiment to be appropriate to write down the responses on prepared answer-sheets. The order of pair presentations was according to the procedure developed by Ross (1934). Ss were instructed to judge the similarity between the members of each possible pair of shapes on a 10 -point scale where it was supposed that 1 means the "almost identical" pair and 10 means the "extremely different" pair. $S$ s were asked that their judgments should be based on the immediate impression of visual shapes. Before collecting data, 10 practice-trials were made so as to familiarize $S$ s with the similarity judgments. The experiment was performed in a class room.

\section{Results}

The mean similarity judgments for each of the 300 pairs of stimuli ranged from 2.35 to 9.54 for Group I and from 1.50 to 9.80 for Group II. The standard deviations ranged from 0.40 to 2.83 and 0.80 to 2.38 for Group I and II, respectively. Spearman's rank-order correlation between the mean similarity judgments for each pair of stimuli of the two groups was significantly high, that is, .92. The two separately obtained means were combined in a final mean similarity judgment, which ranged from 2.19 to 9.44 .

The Kruskal's non-metric analysis was applied to the rank order information of the similarity data. The program was set to stop the iterations for the dimensionality if Stress (a measure of goodnessof-fit) reached $5 \%$ or less and also the iterations were stopped when the minimum Stress greater than 5\% was reached. The iterative procedure was run for Minkowski $r$-metrics of $r=1.0$ (city-block model), 


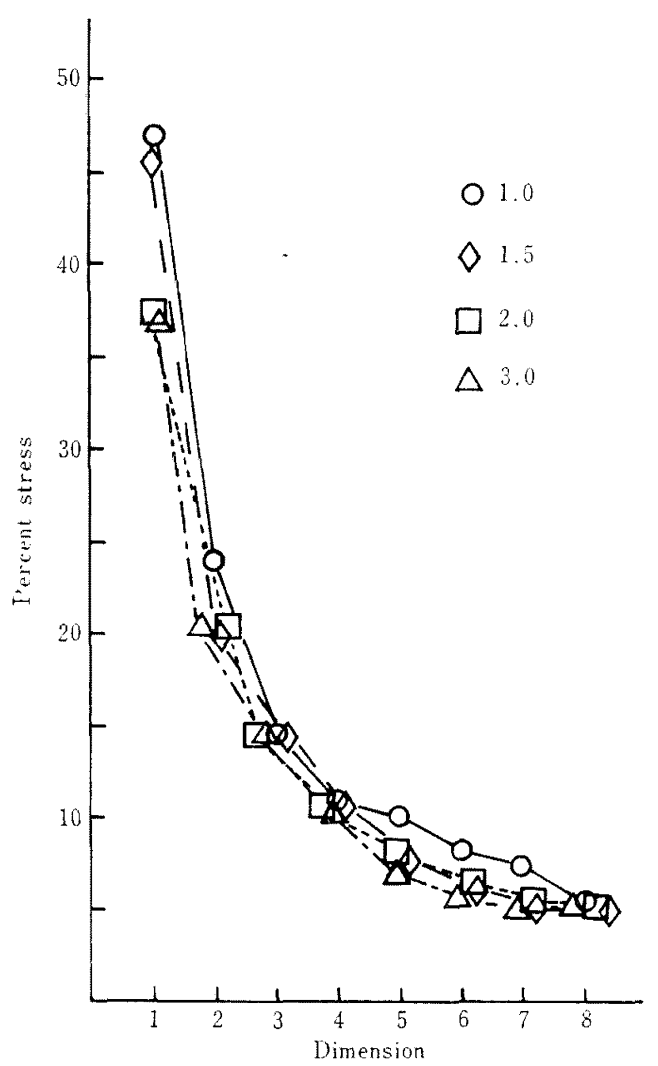

FIG. 2. Stress curves for a few Minkowski in metrics up to eight number of dimensions.

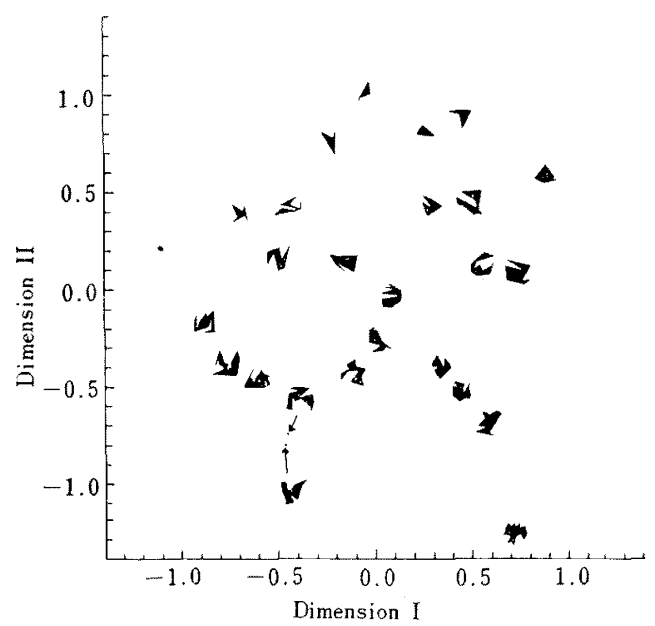

(a) $r=1.5, r=2.0$ (Euclidean model), and $r=$ 3.0. Fig. 2 shows the percent of Stress plotted against the number of dimensions for each metric.

Generally, Stress decreases as the number of dimensions increases. If a certain number of dimensions makes Stress acceptably small, and further increase in the number of dimensions does not reduce Stress noticeably, this number of dimensions will be choosed to be appropriate. Good data sometimes shows an elbow-like curve.

As expected, Fig. 2 shows progressive reduction of Stress as the number of dimensions increases. Fig. 2 also shows that the Stress curves for all solutions drop smoothly with the exception of the curve of $r=1.0$, which shows more or less fluctuations. The similarity data indicates particularly the poorest fit to the city-block model $(r=1.0)$. Among other models, however, no definitive statements are either possible to judge which metric is most appropriate. So that, presently an orthodox Euclidean solution would adequately be accepted, since no convincing advantages were found in other metrics as far as this experiment was concerned. According to the shapes of the Stress curves,

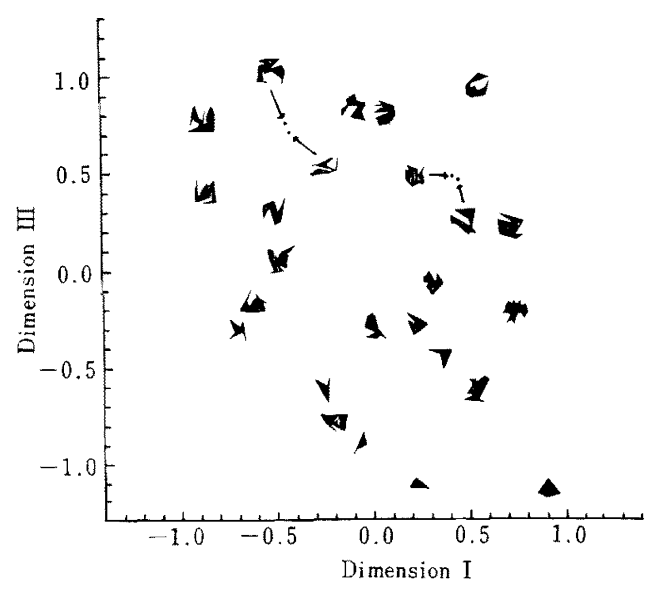

(b)

FIG. 3. The plot of shapes projected on dimension I against dimension II (a), and on dimension I against dimension III (b) $\cdots \cdots$. similarity space. 
and the interpretability of the results, 3 dimensional solution would be accepted, although the measures of Stress were somewhat high, i.e., about $14 \%$ for all solutions. This value suggested that the goodnessof-fit between solution and data was " poor to fair " according to the Kruskal's verbal evaluation.

Three dimensional space for $r=2.0$ (Euclidean) is presented in Fig. 3 as the shapes are projected on the two dimensional transectional space. The features of configuration of the stimuli as shown in Fig. 3 suggest that the first dimension is the dimension of " compactness", though the physical correlates of the dimension such as perimeter, area, perimeter squared divided by area, angular variability, etc. were still remained to be confirmed. The second dimension might be labelled "complexity", because the projected order of stimuli corresponded with the number of turns in the shapes excepting for a few cases. The third dimension was difficult to interpret. However, considering the stimuli locating at the extremes of the dimension, it may be interpreted as a dimension of "symmetry". Stimuli 3 and 5, for example, would seem to be more symmetric than would Stimuli 14 and 17.

As mentioned above, it seems to be rather doubtful whether any meaningful solutions or interpretations are available by further dimensional analysis.

\section{EXPERIMENT II}

\section{Method}

Subjects Participating in the experiment were 40 undergraduate students, 38 males and 2 females, selected from the pool of students in an introductory psychology course at Chiba University.

Stimuli The stimuli were the same as those used in Experiment I.

Scales In a preliminary experiment, adjectives were collected, and then 30-bipolar adjectivescales were selected as being appropriate to the stimuli used here from the collection on referring to other researches with the semantic differential.

On each page of a booklet, the 30 semantic scales were printed originally in Japanese ${ }^{2}$, whose English near-equivalents are shown in Table 1. The polarity of an adjective of each scale and the order of the 30 scales were determined arbitrary. A 7-point rating-form was used.

Procedure Each of the 25 random shapes was projected onto a viewing screen one by one long enough for $S \mathrm{~s}$ to write down their responses on the prepared booklets which contained separate pages for judgments of each shape. The order of presentations of the 25 shapes was determined at random. Ss were required to rate the presented shape on the basis of their immediate impressions of visual forms. $S \mathrm{~s}$ made practice judgments in order to be familiar with the task, before collecting data. The experiment was performed in a class room.

\section{Results}

The rating scores of the shapes were defined on the 7-point scale. The $30 \times 30$ intercorrelation matrix based on the mean rating-scores for each of the shapes was subjected to principal components analysis. The derived 3 factors (accounting for about $80 \%$ of the variance) were orthogonally rotated by Varimax method. The results

2 Semantic scales in Japanese are as follows: 1. Kükyona-Jūjitsushita, 2. Atarashii-Furui, 3. Suiheitekina-Suichokutekina, 4. Inkina-Yookina, 5. Katai-Yawarakai, 6. Shōkyokutekina-Sekkyokutekina, 7. Tanjunna-Fukuzatsuna, 8. Najiminonai-Najiminoaru, 9. Surudoi-Nibui, 10. Futsūno-Fūgawarina, 11. Atatakai-Tsumetai, 12. Danseitekina-Joseitekina, 13. Barabarano-Matomatta, 14. HitaishotekinaTaishotekina, 15. Yukaina-Fuyukaina, 16. Yoi-Warui, 17. Okii-Chiisai, 18. Sukina-Kiraina, 19. OsoiHayai, 20. Anzenna-Kikenna, 21. Jimina-Hadena 22. Anteishita-Fuanteina, 23. Muimina-Yüimina 24. Seitekina-Dōtekina, 25. Yowai-Tsuyoi, 26. Omoshiroi-Tsumaranai, 27. Karui-Omoi, 28. Kinchōshita-Nonbirishita, 29. Utsukushii-Minikui, 30. Akarui-Kurai. 
TABLE 1

Rotated factor matrix

\begin{tabular}{|c|c|c|c|c|c|c|}
\hline & \multirow{2}{*}{\multicolumn{2}{|c|}{ Scales }} & \multicolumn{4}{|c|}{ Factor loadings } \\
\hline & & & Factor I & Factor II & Factor III & Communalities \\
\hline 1 & empty & - full & -011 & 519 & -560 & 577 \\
\hline 2 & new & - old & -834 & 469 & 038 & 915 \\
\hline 3 & horizontal & - vertical & 413 & 248 & 072 & 353 \\
\hline 4 & gloomy & - merry & 144 & 014 & -923 & 866 \\
\hline 5 & hard & - soft & -555 & -579 & -500 & 818 \\
\hline 6 & negative & - positive & 940 & -032 & -255 & 948 \\
\hline 7 & simple & - complex & 123 & -871 & 174 & 831 \\
\hline 8 & unfamiliar & - familiar & -273 & 825 & -377 & 900 \\
\hline 9 & acute & - blunt & -970 & -062 & -113 & 967 \\
\hline 10 & usual & - unusual & 261 & -894 & 186 & 909 \\
\hline 11 & warm & $-\mathrm{cool}$ & 838 & 133 & 424 & 868 \\
\hline 12 & masculine & - feminine & -611 & -574 & 024 & 677 \\
\hline 13 & scattered & - collective & -044 & 929 & -248 & 929 \\
\hline 14 & asymmetric & - symmetric & 065 & 906 & 100 & 815 \\
\hline 15 & pleasant & - unpleasant & 299 & -038 & 886 & 814 \\
\hline 16 & good & - bad & -084 & -422 & 800 & 821 \\
\hline 17 & large & - small & 369 & 207 & 270 & 434 \\
\hline 18 & like & — dislike & -013 & -454 & 779 & 872 \\
\hline 19 & slow & - fast & 889 & 281 & -204 & 918 \\
\hline 20 & safe & - dangerous & 928 & -237 & 159 & 948 \\
\hline 21 & sober & - gay & 901 & -294 & -163 & 939 \\
\hline 22 & stable & - unstable & 634 & -548 & -017 & 717 \\
\hline 23 & unmeaningful & - meaningful & 199 & 142 & -543 & 497 \\
\hline 24 & static & - dynamic & 875 & -081 & -299 & 867 \\
\hline 25 & weak & - strong & 490 & 704 & -097 & 734 \\
\hline 26 & interesting & - uninteresting & -126 & 438 & 728 & 859 \\
\hline 27 & light & - dark & -789 & 034 & 147 & 665 \\
\hline 28 & excitable & - calm & -963 & -173 & -091 & 957 \\
\hline 29 & beautiful & - ugly & -519 & -560 & 458 & 807 \\
\hline 30 & bright & - dark & -270 & -344 & 763 & 735 \\
\hline
\end{tabular}

(Decimal points have been omitted.)

of the analysis were shown in Table 1 .

These resulting factors agree fairly well with those found in semantic differential analyses of other materials such as colors, words, sounds and so on. The first, Factor I, may represent a dimension of activity. Scales having high loadings for this factor are acute-blunt (.97), excitable-calm (.96), positive-negative (.94), dangerous-sale (.93), and gay-sober (.90).
Scales such as fast-slow (.89), dynamicstatic (.88), and cool-warm (.84) have also high loadings for this factor. The first factor extracted accounts for $35.3 \%$ of the total variance. Factor II may be identified as a dimension of potency. Scales being loaded high on this factor are scattered-collective (.93), asymmetricsymmetric (.91), unusual-usual (.89), complex-simple (.89), and unfamiliar- 


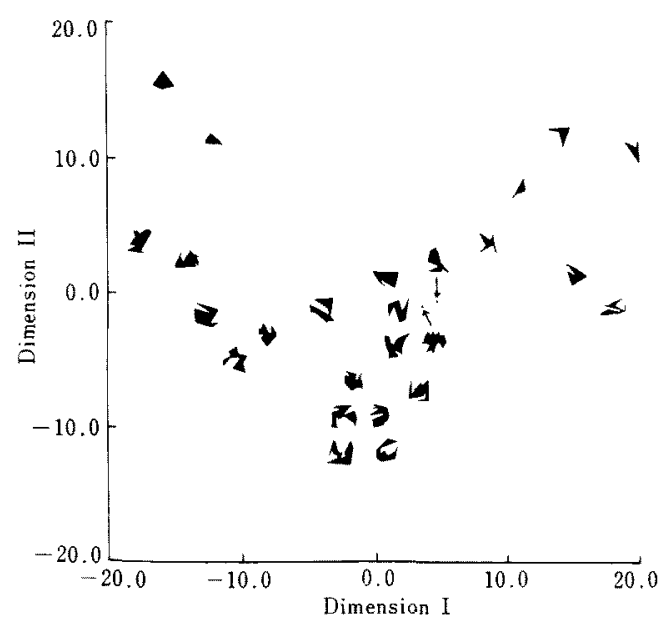

(a)

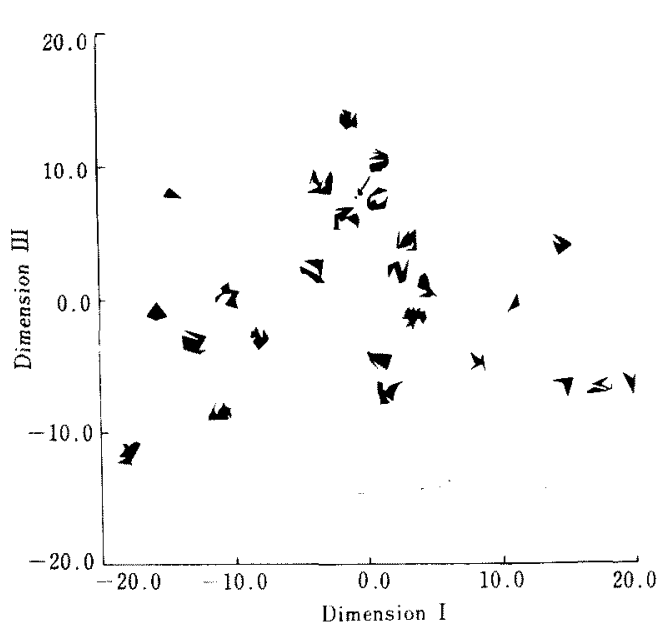

(b)

FIG. 4. The plot of stimulus shapes on dimension 1 against dimension II (a), and on dimension I against dimension III (b) …... semantic space.

TABLE 2

Intercorrelations among the dimensions of different scaling solutions. $R$ and SD denote the Minkowski $r$-metric and the semantic solution, respectively

\begin{tabular}{|c|c|c|c|c|c|c|c|c|c|c|c|c|c|c|c|c|}
\hline & & \multicolumn{3}{|c|}{$\mathrm{R}=1.0$} & \multicolumn{3}{|c|}{$\mathrm{R}=1.5$} & \multicolumn{3}{|c|}{$\mathrm{R}=2.0$} & \multicolumn{3}{|c|}{$\mathrm{R}=3.0$} & \multicolumn{3}{|c|}{$\mathrm{SD}$} \\
\hline & & 1 & 2 & 3 & 1 & 2 & 3 & 1 & 2 & 3 & 1 & 2 & 3 & 1 & 2 & 3 \\
\hline \multirow[t]{3}{*}{$R=1.0$} & 1 & & 10 & 04 & $* * 77$ & 11 & 38 & $* * 91$ & 10 & 05 & $* * 72$ & 15 & 09 & 17 & 09 & 13 \\
\hline & 2 & & & 14 & $* * 64$ & $* 47$ & 09 & 28 & 15 & $* * 76$ & $* 49$ & 05 & $* * 69$ & 32 & $* * 53$ & $* 43$ \\
\hline & 3 & & & & 03 & $* * 77$ & $* * 51$ & 10 & $* * 78$ & $* * 62$ & $* 44$ & $* * 76$ & $* * 53$ & 36 & $* * 64$ & 19 \\
\hline \multirow[t]{3}{*}{$\mathrm{R}=1.5$} & 1 & & & & & 12 & 16 & $* * 77$ & 27 & $* 40$ & $* * 73$ & 28 & 33 & 23 & 30 & 14 \\
\hline & 2 & & & & & & 34 & 05 & $* * 69$ & $* * 90$ & $* 47$ & $* * 71$ & $* * 88$ & 24 & $* * 90$ & 32 \\
\hline & 3 & & & & & & & $* * 65$ & $* * 58$ & $* 40$ & $* * 74$ & $* * 57$ & 05 & 27 & $* 47$ & 10 \\
\hline \multirow[t]{3}{*}{$\mathrm{R}=2.0$} & 1 & & & & & & & & 05 & 25 & $* * 87$ & 06 & 02 & 35 & 27 & 05 \\
\hline & 2 & & & & & & & & & $* 41$ & 34 & $* * 88$ & 32 & 35 & $* * 62$ & 03 \\
\hline & 3 & & & & & & & & & & $* * 63$ & $* 50$ & $* * 91$ & 05 & $* * 87$ & 42 \\
\hline \multirow[t]{3}{*}{$\mathrm{R}=3.0$} & 1 & & & & & & & & & & & 29 & $* 40$ & 23 & $* * 63$ & 15 \\
\hline & 2 & & & & & & & & & & & & 32 & 23 & $* * 62$ & 03 \\
\hline & 3 & & & & & & & & & & & & & 17 & $* * 80$ & $* 46$ \\
\hline \multirow[t]{3}{*}{$\mathrm{SD}$} & 1 & & & & & & & & & & & & & & 07 & 08 \\
\hline & 2 & & & & & & & & & & & & & & & $* 48$ \\
\hline & 3 & & & & & & & & & & & & & & & \\
\hline
\end{tabular}

The sign * and ** denote the significance at .05 level and at .01 level, respectively. (Signs and decimal points have been omitted.) 
familiar (.82). Factor II accounts for $28.0 \%$ of the total variance. Scales having high loadings for Factor III include merry-gloomy (.92), pleasant-unpleasant (.88), good-bad (.80), like-dislike (.78), bright-dark $(.76)$, and interesting-uninteresting (.73). Factor III which accounts for $16.6 \%$ of the total variance is considered as a dimension of evaluation.

The factor scores were estimated for each shape to define the configurations in the semantic space. The configurations of the stimuli in the semantic space is shown in Fig. 4, in which dimensions I, II, and III represent activity, potency, and evaluation dimensions, respectively.

\section{Discussion}

Table 2 gives intercorrelations among the projections of stimuli on the dimensions of the various solutions. In this table, $\mathrm{R}$ and $\mathrm{SD}$ denote the Minkowski $r$-metric and the semantic solution, respectively. The sign* in the table indicates the significant relation at the .05 level and the $\operatorname{sign}^{* *}$ indicates the significance at the .01 level. The table shows that the similarity solutions are highly related each other. However, the activity dimension in the semantic space is not correlated significantly with the similarity dimensions at the .05 level. Nor the evaluation dimension is related significantly with the similarity dimensions at the .01 level. The activity dimension and probably the evaluation dimension are considered as unique dimensions in the semantic space of the shapes used here.

In addition, canonical correlation analysis was applied in order to see the relationship between the semantic space and the similarity space for $r=2.0$ (Euclidean) which is considered as a representative of the similarity solutions. Canonical correlation analysis is a method of obtaining the maximum correlation between linear composites (canonical variates) of the two sets of variates of the same objects. Canon- ical correlation may also be regarded as a method of measuring the amount of variance common to the two sets of variates of the same objects. The weights applied to the original variates in order to obtain the canonical variates indicate the relative contribution of the original variates to each canonical variate. This weight is often called the canonical weight.

The results of the analysis indicate the maximum canonical correlation is .68, and this is significant at the .005 level. Therefore, there is at least one significant way in which the two spaces are related. After the first pair of canonical variates are determined, no further significant combinations seem to exist. The second canonical correlation was .45 , which was not significant at the .05 level, and the third was almost negligible. The test of significance described above is based on Bartlett's $\chi^{2}$ approximation for all canonical correlations. The contribution of each dimension to the significantly related canonical variates may be found in Table 3. In this table the dimension of compactness in the similarity space has remarkably higher weight than the others. On the other hand, the potency dimension has the highest weight of these in the semantic space, though all dimensions in this space have some weights. The squared canonical correlations may be interpreted as the proportion of variance common to their individual canonical variates. The first set of canonical variates shares $46.1 \%$ common variance. The common variance of the second set of variates is only $20.2 \%$ and that of the third

\section{TABle 3}

Contribution of the individual dimensions to the significantly related canonical variates

\begin{tabular}{l|r|l|r}
\multicolumn{2}{c|}{ Similarity space } & \multicolumn{2}{c}{ Semantic space } \\
\hline compactness & .83 & activity & .51 \\
complexity & -.05 & potency & .73 \\
symmetry & .27 & evaluation & .46
\end{tabular}


set of variates is almost zero. The total variance shared by the two spaces is obtained by squaring and summing the canonical correlations and then dividing this sum by three. The result of this computation shows that the total variance shared by the two spaces is $22.1 \%$.

In this study, three dimensions were obtained from the similarity judgments, representing dimensions of compactness, complexity and symmetry. Also three factors were found from the semantic differential, representing dimensions of activity, potency and evaluation. In similar studies (Thomas, 1968; Behrman \& Brown, 1968; Aiken \& Brown, 1969b for the similarity judgments; Elliott \& Tannenbaum, 1963; Oyama \& Haga, 1963 for the semantic differential), almost same number of dimensions was obtained. It is clear from the results that observers are using a variety of stimulus attributes to judge and they seem to give selective attention to three or so attributes in the processing of the tasks. In the case of present study, canonical correlation analysis shows one significant relation, to which the compactness dimension in the similarity space and the potency dimension in the semantic space contribute considerably. The representative scale of the potency dimension may be scattered-collective or asymmetric-symmetric. So that, compactness or symmetry may be a common attribute used to meet the tasks which were performed in this study.

The results of Kruskal's scaling analysis may support the general conclusion that Euclidean spatial presentation gives a favourable model for the complex, unanalyzable stimuli, though no definitive statement is capable of judging which metric is most appropriate. On the other hand, non-Euclidean model (especially city-block model) has been generally considered as the most adequate spatial presentation for the perceptually analyzable stimuli (Attneave, 1950; Hyman \& Well, 1967, 1968; Thomas, 1968).

\section{Conalusion}

In order to examine the relationship between the similarity space and the semantic space of 25 random shapes, two experiments were performed. In Experiment I, subjects rated the similarity between the members of each pair of 25 shapes on a 10-point scale. The Kruskal's nonmetric analysis was applied to the similarity data, and three dimensional solution for $r=2.0$ (Euclidean) was accepted as the representative of the similarity space in this study. The dimensions were labelled as compactness, complexity, and symmetry, respectively. In Experiment II, the same 25 random shapes were judged on the 30 semantic scales. A 7-point ratingform was used. The data were put to principal components analysis. The three factors thus extracted were identified as dimensions of activity, potency, and evaluation, and were found to account for about $80 \%$ of total variance. These two spaces were compared using canonical correlation analysis, which indicated that there was at least one significant relation between them, and total variance shared by them was only $22.1 \%$.

\section{REFERENCES}

Aiken, L. S., \& Brown, D. R. 1969a A spatial analysis of the discriminability of forms in noise. Percept. Psychophys., 5, 171-175.

Alken, L. S., \& Brown, D. R. 1969b Visual form perception: Congruence among spatial configuration. Percept. Psychophys., 5, 155-160.

AikeN, L. S., \& Brown, D. R. 1969c Visual form perception: Task demands and congruence among spatial solution. Percept. Psychophys, 15, 219-220.

Atrneave, F., \& Arnoult, M. D. 1965 The quantitative study of shape and pattern recognition. Psychol. Bull., 53, 221-227.

Behrman, B. W., \& Brown, D. R. 1968 Multidimensional scaling of form: A psychophysical analysis. Percept. Psychophys., 4, 19-25.

Brown, D. R., \& Andrews, H. M. 1968 Visual form discrimination: Multidimensional analy- 
sis. Percept. Psychophys., 3, 401-406.

Er.lot, L. L., \& Tannenbaum, P. H. 1963 Factor-structure of semantic differential responses to visual forms and prediction of factor score from structural characteristics of stimulus shapes. Amer. J. Psychol., 76, 589-597.

Hyman, R., \& Welt, A. 1967 Judgments of similarity and spatial models. Percept. Psychophys., 2, 233-248.

Hyman, R., \& Well, A. 1968 Perceptual separability and spatial models. Percept. Psychophys., 3, 161-165.

Kruskal, J. B. 1964a Multidimensional scaling by optimizing goodness of fit to a nonmetric hypothesis. Psychometrika, 29, 1-27.

Kruskal, J. B. 1964b Nonmetric multidimensional scaling: A numerical method. Psychometrika, 29, 115-129.

Oyama, T., \& HagA, J. 1963 Common factors between figural and phonetic symbolism. Psychologia, 6, 131-144.

Ross, R. T. 1934 Optimum order for the presentation of pairs in the method of paired comparison. 7. educ. Psychol., 25, 375-382.

Thomas, H. 1968 Spatial models and multidimensional scaling of random shapes. Amer. 7 . Psychol., 81, 551-558.

(Received Dec. 13, 1971) 